\title{
Method for measurement of transition probabilities by laser-induced breakdown spectroscopy based on CSigma graphs. Application to Ca II spectral lines
}

\author{
J. A. Aguilera ${ }^{a, b *}$, C. Aragón ${ }^{a, b}$, J. Manrique ${ }^{c}$ \\ ${ }^{a}$ Departamento de Física, Universidad Pública de Navarra, Campus de Arrosadía, E-31006 Pamplona, Spain \\ ${ }^{b}$ Institute for Advanced Materials (INAMAT), Public University of Navarre, Campus de Arrosadía, \\ E-31006 Pamplona, Spain \\ ${ }^{c}$ Facultad de Farmacia, Universidad CEU San Pablo, Urbanización Montepríncipe, Boadilla del Monte, \\ E-28668 Madrid, Spain
}

\begin{abstract}
We propose a method for determination of transition probabilities by laser-induced breakdown spectroscopy that avoids the error due to self-absorption. The method relies on CSigma graphs, a generalization of curves of growth which allows including several lines of various elements in the same ionization state. CSigma graphs are constructed including reference lines of an emitting species with well-known transition probabilities, together with the lines of interest, both in the same ionization state. The samples are fused glass disks prepared from small concentrations of compounds. When the method is applied, the concentration of the element of interest in the sample must be controlled to avoid the failure of the homogeneous plasma model. To test the method, the transition probabilities of $9 \mathrm{Ca}$ II lines arising from the $4 \mathrm{~d}, 5 \mathrm{~s}, 5 \mathrm{~d}$ and 6 s configurations are measured using Fe II reference lines. The data for 5 of the studied lines, mainly from the $5 \mathrm{~d}$ and 6 s configurations, had not been measured previously.
\end{abstract}

\section{Keywords:}

Transition probabilities; Laser induced breakdown spectroscopy; CSigma graph, Ca II

*Corresponding author.

Tel: +34-948169579

Fax: +34-948169565.

E-mail address: j.a.aguilera@unavarra.es 


\section{Introduction}

Accurate data for transition probabilities of spectral lines of neutral and ionized atoms are necessary for the diagnostics of different types of plasmas, including astrophysical, laboratory, fusion and industrial plasmas. From a theoretical point of view, transition probability calculations are very sensitive to the interaction schemes used, so experimental data allow testing the validity of the atomic structure models. However, for many atoms and ions, the available data are scarce or suffer from high uncertainty. Ionized calcium has been used in astrophysical observations from galaxies, interstellar gas clouds, stars [1, 2] and meteors [3], mainly the relevant $\mathrm{H}$ and $\mathrm{K}$ lines. Laboratory experiments involving transitions between the low energy levels of the Ca II have been recently used to study the optical frequency standard and quantum information processing [4, 5]. Previous values of transition probabilities of Ca II were firstly obtained by Gallagher [6] for three spectral lines arising from the $4 p$ level introducing atomic vapours into an argon discharge. Branching ratios precision measurements were performed for the same lines by Gerritsma et al. [7] with a single calcium ion suspended in a linear Paul trap. The only experimental measurements found in the literature for other lines were carried out by Andersen et al. [8] using the beam-foil technique to obtain oscillator strengths including 4d, 5s and $5 \mathrm{~d}$ levels in the Ca II spectrum. Although there is a shortage of experimental data, theoretical calculation of transition probabilities of Ca II has received a considerable effort due to its quasi-hydrogenic nature. There is good agreement between works only related to the lower levels $[9,10]$ whilst several theoretical approaches have been used with different agreement for calculations including higher energy levels. Biemont [11] obtained new oscillator strengths for the potassium isoelectronic sequence by Hartree-Fock wavefunctions. A relativistic pseudopotential approach was employed by Hafner and Schwarz [12] whereas a core polarization potential was tested by Laughlin [13]. Meléndez et al. [14] concluded, by using the Thomas-Fermi-Dirac central potential method, that polarization interaction can affect the oscillator strength by up $12 \%$. Mitroy and Zhang [15] employed the semiempirical approach whilst a systematic study of the ionized calcium was carried out by Safronova and Safronova [16] using a relativistic high order method. In order to test the different theoretical methods used, a lack of experimental measurements remains, where self-absorption is one of the most important sources of error.

Laser-induced breakdown spectroscopy (LIBS) has been increasingly accepted as a technique suitable for the measurement of atomic data, including transition probabilities [17] and Stark broadening and shift parameters [18]. Two approaches were used in early works for the determination of transition probabilities by LIBS [17]. One of them was based on the measurement of branching ratios from the relative intensities in the LIBS spectra. The absolute transition probabilities were then obtained using experimental lifetimes and line-strength sum rules. In the second approach, a Boltzmann plot is constructed using lines with known transition probabilities from the literature. Then, the absolute transition probabilities of the lines of interest are deduced from the Boltzmann plot and the measurement of the corresponding line intensity. This method relies on the existence of local thermodynamic equilibrium in the plasma, whose excitation temperature is determined. The two methods described require that the lines used are emitted in optically thin conditions. Generally, this requirement is not easily accomplished in laser-induced plasmas, which are sources characterized by a high density. The usual method to ensure optically thin conditions consists in reducing the concentration of the emitting element in the sample and performing an estimation of self-absorption. However, the suitable concentration is different for each of the lines of interest, as self-absorption depends on the line intensity and line width. In previous works [19, 20], our group has determined transition probabilities by LIBS using a method that avoids self-absorption, based on the measurement and calculation of curves of growth. This method requires the knowledge of transition probabilities and Stark widths for a group of lines of the atom or ion investigated. From the curves of growth of these lines, the plasma is characterized by a small set of parameters and then the oscillator strengths of the lines of interest are determined by fitting their experimental curves of growth. A disadvantage of this method is the need for a relatively wide set of samples of known concentrations. Also, characterization is performed from several curves of growth of different lines, which implies a certain complexity in the fitting process.

In a recent work [21], our group has introduced $C \sigma$ graphs as generalized curves of growth, which allow including several lines of various elements in the same ionization state at different concentrations. In [21], $C \sigma$ graphs are proposed as a new approach for plasma characterization in LIBS. In the present work, we describe a method for measurement of transition probabilities based on $C \sigma$ graphs. This method aims at avoiding selfabsorption in the measurement and overcoming the drawbacks of our previous approach based on conventional curves of growth. To test the method, transition probabilities of Ca II lines are measured and the results are compared to available values in the literature. 


\section{Measurement procedure}

\subsection{Co graphs}

The method for plasma characterization based on $C \sigma$ graphs was described in detail in our previous work [21], so only the main definitions and equations are recalled here. By integrating the radiative transfer equation for a homogeneous plasma in local thermodynamic equilibrium, the following expression is obtained for the wavelength-integrated line intensity

$$
I=\beta A L_{P} \int_{\text {line }}\left(1-e^{-\tau(\lambda)}\right) d \lambda=\beta A L_{P} \int_{\text {line }}\left(1-e^{-k^{\prime}(\lambda) l}\right) d \lambda,
$$

where $A$ is the transverse area of the region of the plasma whose emission is detected, $\beta$ is the instrumental factor of the system, $L_{P}=L_{P}\left(\lambda_{0}, T\right)$ is the Planck radiance of a blackbody, considered constant in the integration over the line profile and calculated at the plasma temperature $T$ and at the central wavelength $\lambda_{0}$ of the transition, $\tau(\lambda)$ is the optical depth, $k^{\prime}(\lambda)$ is the effective absorption coefficient and $l$ is the length of the plasma along the lineof-sight. Two assumptions are made at this point: the laser-induced plasma is formed only by neutral atoms and singly-charged ions and the stoichiometry in the ablation process is maintained. With these assumptions, the optical depth may be factorized as follows

$$
\tau(\lambda)=C N l k_{t} r_{i} V(\lambda)
$$

where $C$ is the concentration of the emitting element in the sample expressed as atomic fraction (at/at), $N$ is the total density in the plasma and $V(\lambda)$ is the line profile, which is described by Voigt profile. The remaining two factors in Eq. (2) have been defined as follows. On one side, the coefficient $k_{t}(T)$ for a transition, dependent on the atomic data of the transition and the temperature, is defined by

$$
k_{t}=\frac{e^{2} \lambda_{0}^{2}}{4 \varepsilon_{0} m c^{2}} f \frac{g_{i} e^{-\frac{E_{i}}{k T}}}{U_{\alpha}^{z}(T)}\left(1-e^{-\frac{E_{k}-E_{i}}{k T}}\right),
$$

where $e$ is the elementary charge, $\varepsilon_{0}$ is the permittivity of free space, $m$ is the electron mass, $c$ is the speed of light in vacuum, $f$ is the transition oscillator strength, $g_{i}$ is the degeneracy of the lower energy level, $E_{i}, E_{k}$ are the energies of the lower and upper energy levels respectively, $k$ is the Boltzmann's constant, $T$ is the absolute temperature, and $U_{\alpha}^{z}(T)$ is the partition function of the emitting species (element $\alpha$ at ionization state $z$ ). On the other side, the ionization factor $r_{i}\left(T, N_{e}\right)$, dependent on electron density $N_{e}$ and temperature, is defined as

$$
\begin{array}{ll}
r_{i}=\frac{1}{1+S^{10}} & \text { for neutral atoms } \\
r_{i}=\frac{S^{10}}{1+S^{10}} & \text { for singly-charged ions. }
\end{array}
$$

In these equations, $S^{10}\left(T, N_{e}\right)$ is the ratio between the density of singly-charged ions $N_{\alpha}^{1}$ and that of neutral atoms $N_{\alpha}^{0}$, which is provided by the Saha equation

$$
S^{10}\left(T, N_{e}\right)=\frac{N_{\alpha}^{1}}{N_{\alpha}^{0}}=\frac{2 U_{\alpha}^{1}}{N_{e} U_{\alpha}^{0}}\left(\frac{m k T}{2 \pi \hbar^{2}}\right)^{3 / 2} \exp \left(-\frac{E_{\infty}^{0}-\Delta E_{\infty}^{0}}{k T}\right),
$$

where $\hbar=h / 2 \pi, E_{\infty}^{0}$ is the ionization energy and $\Delta E_{\infty}^{0}$ is the correction thereof due to interactions in the plasma.

To construct a $C \sigma$ graph from several emission lines of various elements in the same ionization state, a line cross section $\sigma_{l}\left(T, N_{e}\right)$ is calculated for each spectral line, defined by 


$$
\sigma_{l}=k_{t} r_{i} \frac{1}{\Delta \lambda_{L}}
$$

where $\Delta \lambda_{L}$ is the Lorentzian width of the line, which is obtained from the electron density and the Stark width, assuming that the main broadening mechanism in the plasma is the Stark effect. The $C \sigma$ graph has abscissas and ordinates defined by the following relation

$$
\frac{I}{L_{P} \Delta \lambda_{L}}=f\left(C \sigma_{l}\right) .
$$

In our previous work [21] it was shown that, when lines of different widths are included in the $C \sigma$ graph defined by Eq. (7), the data follow a smooth behaviour for low to moderate values of the abscissa and Lorentzian widths higher than $0.01 \AA$ (damping ratios $a>1$ ). Moreover, a common linear limit with slope given by the product $\beta A \times N l$ exists as $C \sigma_{l} \rightarrow 0$ and self-absorption tends to disappear. A consequence of this behaviour in experimental $C \sigma$ graphs is that, taking into account the errors, the data for different Lorentzian widths form a single curve within a good approximation. From Eq. (1), using Eq. (2) for the optical depth, numerical integration allows to calculate the ordinate of a $C \sigma$ graph for the same values of the abscissa plotted in the experimental graph. In this calculation, the values of $T, N_{e}$ are determined independently (by the Boltzmann plot and Stark broadening methods), whereas typical initial values of $\beta A, N l$ are introduced. The fitting of the experimental $C \sigma$ graph to such calculated $C \sigma$ curves provides the parameter $\beta A$, the columnar density $\mathrm{Nl}$ and a value of the temperature $T$, which is very similar to that resulting from the Boltzmann plot. These parameters, together with the electron density $N_{e}$, characterize the plasma for the corresponding ionization state. On the other side, from the four parameters, a theoretical $C \sigma$ curve may be calculated that characterizes the LIBS system for a given ionization state. In the present work, $C \sigma$ graphs are used for the determination of transition probabilities of Ca II lines. To this aim, a set of reference Fe II lines, whose transition probabilities are known, are used to construct an accurate $C \sigma$ graph. Table 1 lists the Fe II lines used, together with their atomic data. The transition probabilities have been taken from the compilation by Fuhr and Wiese [22]. The Ca II lines of interest are listed in Table 2, with their transition probabilities and oscillator strengths obtained from the NIST Atomic Database [23]. The Stark widths for most of the Fe II and Ca II lines have been measured in previous works of our group [24-27]. To illustrate $C \sigma$ graphs and their application for transition probability determination, we have calculated one of such graphs including the Fe II reference lines of Table 1 and the Ca II lines numbered 1-5 in Table 2. This theoretical $C \sigma$ graph has been obtained for typical values of the electron density, temperature and columnar density in the plasma. Also, typical values of the Fe and Ca concentrations found in fused glass samples have been used in this example. The resulting $C \sigma$ curve is shown in Fig. 1, where the points in the curve corresponding to Ca II lines are numbered as in Table 2. The linear limit for $C \sigma_{l} \rightarrow 0$ is also displayed in the figure. Figure 1 shows that, in spite of the different widths of the Fe II and Ca II lines, in the range $0.036-0.052 \AA$ for Fe II and $0.49-0.77 \AA$ for Ca II, the data follow a very smooth behaviour, which allows assuming that they belong approximately to a unique $C \sigma$ curve.

\subsection{Model limit}

In the theoretical framework described in the previous subsection, a homogeneous model of the laserinduced plasma is used in the integration of the radiative transfer equation performed to obtain the line intensities. However, laser-induced plasmas are in fact inhomogeneous sources having spatial gradients of their characteristic parameters. As a consequence, as described in [21], $C \sigma$ curves calculated using the model of homogeneous plasma will not be able to provide accurately the ordinate of $C \sigma$ graphs for experimental data corresponding to intense lines or high concentrations. The approach used to account for this limitation consists in defining a limit for the validity of the homogeneous model. To obtain this limit, we previously define a line optical depth $\tau_{l}\left(T, N_{e}\right)$ for each data of a $C \sigma$ graph, which may be expressed as

$$
\tau_{l}=C N l \sigma_{l} .
$$

To check if the data exceed the model limit, the following quantity is evaluated

$$
\tau_{l} \Delta \lambda_{L}=C N l k_{t} r_{i} .
$$


Indeed, the product $\tau_{l} \Delta \lambda_{L}$ increases as the columnar density $N l$ increases, the concentration increases, or the line considered has a higher intensity (high $\left.k_{t} r_{i}\right)$. The model limit $\left(\tau_{l} \Delta \lambda_{L}\right)$ lim is established experimentally by the convergence of $\chi^{2}$ in the fitting of the $C \sigma$ graph as the data with higher values of $\tau_{l} \Delta \lambda_{L}$ are successively eliminated. In our previous work [21], the model limit obtained was $\left(\tau_{l} \Delta \lambda_{L}\right)_{\lim } \cong 0.1 \AA$ for a laser-induced plasma generated in similar experimental conditions to those of the present work.

\subsection{Procedure for determination of transition probabilities}

Once we have obtained a $C \sigma$ graph including reference lines as well as the lines of interest, with all its data fulfilling the condition for model validity, the procedure for determining the transition probabilities is straightforward. From the fitting of the data corresponding to the reference lines, the characteristic plasma parameters and, from them, the $C \sigma$ curve are obtained. As the abscissa of the data depends on the oscillator strength $f$ through the line cross section $\sigma_{l}$, an error in the value of $f$ shifts the abscissa in the graph, taking the point out of the curve. The new value of the oscillator strength is then determined by an iterated process which fits the abscissa of the point to that of the numerically-calculated reference curve. It is also possible, using various samples, to include in this procedure data corresponding to several concentrations for the same line. However, this approach assumes the absence of matrix effects in the formation of the plasma, so that the plasma parameters are the same for the different samples. In the case where no previous data are available for the transition probability, the procedure is initialized using an arbitrary value for the oscillator strength.

\section{Experiment}

The experimental system is the same used in previous works [20, 24], so it is only described briefly. Laserinduced plasmas are generated by focusing a Nd:YAG laser (wavelength $1064 \mathrm{~nm}$, pulse energy $60 \mathrm{~mJ}$, pulse width $4.5 \mathrm{~ns}$ ) at right angles onto the sample surface, in air at atmospheric pressure. The focusing lens has a focal length of $126 \mathrm{~mm}$ and the lens to sample distance is $122 \mathrm{~mm}$. The plasma emission is collected at a small angle from the laser beam direction by a pair of flat and concave mirrors, which form a 1:1 image on the entrance slit of the spectrometer (Czerny-Turner, focal length $0.75 \mathrm{~m}$, gratings of 3600 and 1200 lines $\mathrm{mm}^{-1}$ ). The instrumental width obtained with the grating of 3600 lin $\mathrm{mm}^{-1}$, used for the measurement of $C \sigma$ graphs, is $0.135 \AA$. The detector is an intensified charge-coupled device having 1200x256 effective pixels. The spectral efficiency of the system has been measured using calibrated tungsten and deuterium lamps. During the measurements, the emission of 100

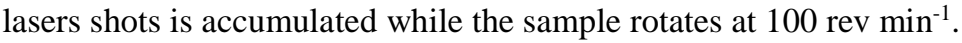

The samples are fused glass disks, prepared from pure compounds by fusion in a lithium borate mixture. In a previous work [26], it was shown that fused glass samples are advantageous for the measurement of Stark widths by LIBS, as the spectra show high line-to-continuum ratios, so that small concentrations in the sample may be used. In the present work, the samples have been prepared from high purity $\mathrm{CaCO}_{3}$ and $\mathrm{Fe}_{2} \mathrm{O}_{3}$. The content of the compounds in the samples has been selected to produce the desired Fe and Ca concentrations, expressed in atomic fraction (at/at) and shown in Table 3.

\section{Results and discussion}

\subsection{Plasma characterization}

The plasma emission has been detected using a time gate with a delay of $1.6 \mu$ s from the laser pulse and a width of $0.4 \mu \mathrm{s}$. A home-made computer program based on least-squares fitting is used to obtain the line intensities and widths from the measured spectra. The electron density of the laser-induced plasma has been measured from the Stark broadening of the $\mathrm{H}_{\alpha}$ line by the same procedure described in previous works [24-27]. The electron density obtained is $(1.78 \pm 0.2) \times 10^{17} \mathrm{~cm}^{-3}$, the value for the three samples used being the same within the statistical error. The parameters $\beta A$ and $\mathrm{Nl}$ have been determined from the Fe II data of the $C \sigma$ graph shown in Fig. 2, measured using sample A. The error bars in all the experimental plots in this work represent the standard deviation of the average of five measurements at different positions in the sample. The Fe content of sample A is large enough to produce a noticeable self-absorption of the Fe II lines with higher line cross section $\sigma_{l}$, as can be seen in the plot of Fig. 2, where the linear limit is displayed. The self-absorption of the more intense Fe II lines allows to obtain the $\beta A$ and $\mathrm{Nl}$ parameters from the fitting of the experimental data to calculated $C \sigma$ curves. The Boltzmann plot shown in Fig. 3 has also been constructed using the less intense Fe II lines, which show a negligible selfabsorption in the plot of Fig. 2. The temperature $T$ determined from the slope of the Boltzmann plot is $13500 \pm$ $250 \mathrm{~K}$. As explained in our previous work [21], the plasma temperature is also deduced from the $C \sigma$ graph, as a wrong temperature value results in a shift of the temperature-dependent abscissas of the data, which increases the $\chi^{2}$ of the fitting to a $C \sigma$ curve. Thus, an iterated process leads to a temperature value which is the same as that resulting from the Boltzmann plot within the experimental error. Once the temperature is known, the fitting of the 
Fe II data in the plot in Fig. 2 provides the columnar density $N l=(2.5 \pm 0.4) \times 10^{20} \mathrm{~m}^{-2}$. The $\beta A$ parameter is also determined from the fitting, which completes the characterization of the plasma, allowing also to plot the final $C \sigma$ curve, shown in Fig. 2.

As can be seen in Fig. 2, the Fe II data of the $C \sigma$ graph show relatively small deviations from the $C \sigma$ curve, which implies that the experimental errors are small and, more importantly, that the reported uncertainties in the transition probabilities of the Fe II lines are likely overestimated. The result is that accurate $C \sigma$ graphs may be obtained using the Fe II lines, providing a precise characterization of the plasma by the set of plasma parameters and the $C \sigma$ curve.

\subsection{Determination of transition probabilities}

In the plot of Fig. 2, obtained using sample A, we notice that the data corresponding to the Ca II lines undergo high deviations from the $C \sigma$ curve, especially for the more intense lines at $3179.331 \AA$ and $3158.869 \AA$, numbered 3 and 4 respectively in Table 2 . This deviations, which could be initially attributed to the errors of the transition probabilities, are actually due to a greater extent to the failure of the homogeneous plasma model. To verify this, we have obtained the values of $\tau_{l} \Delta \lambda_{L}$ for the lines $1-5$, calculated with the Ca concentrations of samples A and B. The results are listed in Table 4, where we can see that, for sample A and the more intense Ca II lines 3, 4 and 1, the values of $\tau_{l} \Delta \lambda_{L}$ are over $0.1 \AA$, whereas for the weaker lines 2 and 5 the values are lower. According to the discussion in subsection 2.2, the data exceeding the model limit are not well described by the homogeneous plasma model and should not be included in $C \sigma$ graphs. The solution to this problem consists in using a sample with a lower Ca concentration. We have checked that, as the Ca concentration is decreased, the data for the more intense lines 3 and 4 gradually approach the $C \sigma$ curve. This behavior converges for these lines as the Ca concentration approaches that of sample B. In Table 4 we see that, for the smaller concentration of sample $\mathrm{B}$, the value of $\tau_{l} \Delta \lambda_{L}$ is clearly below $0.1 \AA$ even for the more intense lines 3 and 4 . Therefore, we estimate the model limit in our experiment as $\left(\tau_{l} \Delta \lambda_{L}\right)_{\lim } \cong 0.1 \AA$, in agreement with the value found in our former work [21]. Figure 4a shows the $C \sigma$ graph obtained with sample B. As can be seen, according to the low Ca concentration in the sample, self-absorption is not noticeable in the graph. Thus, the $C \sigma$ curve is linear and the fitting parameter is the slope $\beta A \times N l$. In Fig. 4a, we also notice that the data for the Ca II lines 3, 4 and 1 are close to the $C \sigma$ curve. The positions of the Ca II data are best seen in Fig. $4 \mathrm{~b}$, where the Fe II data have been removed. The transition probabilities for the Ca II lines have been determined from the data in this graph by the procedure described in subsection 2.3. From the figure, we may notice that the new values will be in good agreement with the data in the NIST Atomic Spectra Database, used to construct the graph. As the $C \sigma$ graphs used in the final measurements are linear or show only weak self-absorption, the statistical error of the resulting transition probability is estimated as the quadratic combination of the standard deviation of the ordinate of the Ca II data and the standard deviation of slope $\beta A \times N l$. For the set of lines studied, the statistical error is lower than $3 \%$. It is worth noting that, for a curve showing self-absorption as that of Fig. 2, the standard deviation of the product $\beta A \times N l$ is much lower than those of the parameters $\beta A$ and $N l$. For the plot of Fig. 4, the standard deviation of $\beta A \times N l$ is only $0.6 \%$ and a standard deviation of about $1 \%$ is typical for this parameter.

The observation in the spectra of the weaker Ca II lines 6-9, which arise from the $5 \mathrm{~d}$ and 6 s configurations, requires to increase the $\mathrm{Ca}$ concentration in the sample. Therefore, sample $\mathrm{C}$ (see Table 3 ) has been used to obtain the $C \sigma$ graph for these lines (not shown), including the same reference Fe II lines listed in Table 1 . In spite of the higher Ca concentration, the data in the graph are below the model limit for these weaker lines. As will be seen in the next subsection, the values obtained for the transition probabilities show in this case significant differences with the theoretical values reported in the literature. On the other side, the resonance Ca II lines have not been included in the study because, due to their high intensity, the model limit imposes that the Ca concentration in the sample must be reduced to extremely low values, which spoils the accuracy of the measurements.

\subsection{Results for transition probabilities}

The experimental transition probabilities obtained are shown in Table 5, together with previous results from the literature. The error of the transition probability displayed in the table is the total experimental error. The sources of error considered include the error of the spectral efficiency, the uncertainty in the measurement of the intensity of Ca II lines, the remaining error due to the plasma inhomogeneity as well as smaller errors as those due to the uncertainty in the determination of the plasma temperature, the error of the concentration in the sample and the statistical error. Combining these errors, we have estimated the total error of our measurements to be $12 \%$ for the lines from the $6 \mathrm{~s}$ and $5 \mathrm{~d}$ configurations and $7 \%$ for the lines from the $5 \mathrm{~s}$ and $4 \mathrm{~d}$ configurations, with the exception of the line at $3181.275 \AA$, for which the error is increased to $12 \%$ due to its overlapping with the line at 3179.331 A. The only available experimental results found in the literature for the transitions investigated in this 
work were performed by Andersen et al. [8]. The experimental and theoretical values for the transitions originating from the $3 \mathrm{p}^{6} 5 \mathrm{~s}$ configuration reported in the references agree to within $7 \%$ with our results. Similar agreement is obtained for lines arising from the $3 \mathrm{p}^{6} 4 \mathrm{~d}$ configuration, except for the one at $3181.275 \AA$ that only can be compared to theoretical calculations with values around $20 \%$ lower. Only one experimental result is given for the other lines in Table 5, corresponding to $2113 \AA$, a result corresponding to the overlapping of two lines, at 2112.757 and $2113.146 \AA$, with a value $55 \%$ higher than ours. The calculations for those lines arising from the $3 p^{6} 6 \mathrm{~s}$ and $3 p^{6} 5 \mathrm{~d}$ configurations apply different theoretical approaches achieving an agreement better than $10 \%$. Nevertheless discrepancies are noticeable by comparing with our results, showing values which are about $60 \%$ and $90 \%$ higher for the lines arising from $6 \mathrm{~s}$ and $5 \mathrm{~d}$ levels respectively. Several theoretical calculations results without fine splitting approach are listed in Table 6 for comparison with our statistically-weighted measurements. The same behaviour is observed for these values, showing discrepancies lower than $8 \%$ for transitions from $5 \mathrm{~s}$ and $4 \mathrm{~d}$ upper levels and values around $60 \%$ and $90 \%$ higher than ours from $6 \mathrm{~s}$ and $5 \mathrm{~d}$ levels respectively.

\section{Conclusions}

A new method for determination of transition probabilities by laser-induced breakdown spectroscopy is proposed. The measurements are performed by means of $C \sigma$ graphs constructed starting from several reference lines with well-known transition probabilities, which provides accuracy to the results. The use of $C \sigma$ graphs and curves allows avoiding the systematic error due to self-absorption, whereas the effect of plasma inhomogeneity is controlled through the selection of the concentration in the sample based on a model limit. We expect that the combination of this method with the use of fused glass samples, which lead to spectra showing increased line-tocontinuum ratios and may be prepared from many different compounds, will allow the measurement of transition probabilities of other atoms and ions of interest.

\section{Acknowledgements}

This work has been supported by the project FIS2011-29521 of the Spanish Ministerio de Economía y Competitividad.

\section{References}

[1] Welty DE, Morton DC, Hobbs LM. A high-resolution survey of interstellar Ca II absorption. Astrophys J Suppl Ser 1996;106: 533-562.

[2] Nestor DB, Pettini M, Hewett PC, Rao S, Wild V. Measurements of Ca II absorption, metals and dust in a

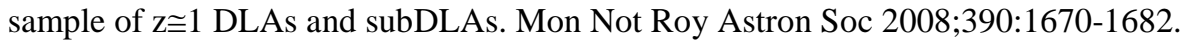

[3] Babadzhanov PB, Kokhirova GI. The concentration of calcium ions and of free electrons in a meteor coma. Astron Astrophys 2004; 424: 317-323.

[4] Chwalla M, Benhelm J, Kim K, Kirchmair G, Monz T, Riebe M, et al.. Absolute Frequency Measurement of the ${ }^{40} \mathrm{Ca}^{+} 4 \mathrm{~s}^{2} \mathrm{~S}_{1 / 2}-3 \mathrm{~d}^{2} \mathrm{D}_{5 / 2}$ Clock Transition. Phys Rev Lett 2009; 102:0230021-0230024.

[5] Hendricks RJ, Sorensen JL, Champenois C, Knoop M, Drewsen M. Doppler cooling of calcium ions using a dipole-forbidden transition. Phys Rev A 2008; 77:0214011-0214014.

[6] Gallagher A. Oscillator Strengths of Ca II, Sr II, and Ba II. Phys Rev 1967; 157:24-30.

[7] Gerritsma R, Kirchmair G, Zahringer F, Benhelm J, Blatt R, Roos CF. Precision measurement of the branching fractions of the $4 \mathrm{p}^{2} \mathrm{P}_{3 / 2}$ decay of Ca II. Eur Phys J D 2008; 50: 13-19.

[8] Andersen T, Desesquelles J, Jessen KA, Sorensen G. Measurements of atomic lifetimes for neutral and ionized calcium. J Quant Spectrosc Radiat Transf 1970; 10: 1143-1150.

[9] Vaeck N, Godefroid M, Fischer CF, Core-valence correlation effects on E1 and E2 decay rates in Ca+. Phys Rev A 1992; 46: 3704-3716.

[10] Brage T, Fischer CF, Vaeck N, Godefroid M, Hibbert A. Core Polarization in Ca I and Ca II. Phys Scr 1993; 48: 533-545.

[11] Biemont E. Cancellation effects and trends of oscillator strengths in the potassium isoelectronic sequence. Physica B \& C 1976; 81:158-180.

[12] Hafner P, Schwarz WHE. Atomic transition probabilities from the relativistic pseudopotential approach. J Phys B: At Mol Opt Phys 1978;11:2975-2999.

[13] Laughlin C. On the accuracy of the Coulomb Approximation and a Model-Potential Method for Atomic Transition Probabilities in Alkali-like Systems. Phys Scr 1992; 45: 238-245.

[14] Melendez M, Bautista MA, Badnell NR. Atomic data from the IRON project - LXIV. Radiative transition rates and collision strengths for CaII. Astron Astrophys 2007; 469: 1203-1209.

[15] Mitroy J, Zhang JY. Long range interactions of the Mg+ and Ca+ ions. Eur Phys J D 2008; 46: 415-424. 
[16] Safronova MS, Safronova UI. Blackbody radiation shift, multipole polarizabilities, oscillator strengths, lifetimes, hyperfine constants, and excitation energies in Ca+. Phys Rev A 2011; 83:0125031-01250317.

[17] Ortiz M, Campos J, Mayo R, Blagoev K, Malcheva G. Possibility of LIBS for transition probabilities determination. Proc of SPIE 2005;5830:226-30.

[18] Konjević N, Ivković M, Jovićević S. Spectroscopic diagnostics of laser-induced plasmas, Spectrochim Acta Part B 2010; 65: 593-602.

[19] Manrique J, Aguilera JA, Aragón C. Determination of transition probabilities by laser-induced breakdown spectroscopy with curve-of-growth measurements. J Quant Spectrosc Radiat Transfer 2011; 112:85-91.

[20] Manrique J, Aguilera JA, Aragón C. Transition probabilities of Ni II spectral lines measured by laser induced breakdown spectroscopy. J Quant Spectrosc Radiat Transfer 2013; 120:120-124.

[21] Aragón C, Aguilera, JA. CSigma graphs: A new approach for plasma characterization in laser-induced breakdown spectroscopy. J Quant Spectrosc Radiat Transfer 2014; 149:90-102.

[22] Fuhr JR,Wiese WL. A critical compilation of atomic transition probabilities for neutral and singly ionized iron. J Phys Chem Ref Data 2006;35:1669-1809.

[23] Kramida A, Ralchenko Yu, Reader J and NIST ASD Team 2014, NIST Atomic Spectra Database (ver. 5.2), [Online]. Available: http://physics.nist.gov/asd. National Institute of Standards and Technology, Gaithersburg, MD.

[24] Aragón C, Vega P, Aguilera JA. Stark width measurements of Fe II lines with wavelengths in the range 260-300 nm. J Phys B:At Mol Opt Phys 2011;44: 055002 (7p).

[25] Aguilera JA, Manrique J, Aragón C. Stark width measurements of FeII lines with wavelengths in the range 230-260 nm. J Phys B At Mol Opt Phys 2011;44:245701(6p).

[26] Aragón C, Aguilera JA, Manrique J. Measurement of Stark broadening parameters of FeII and NiII spectral lines by laser induced breakdown spectroscopy using fused glass samples. J Quant Spectr Radiat Transfer 2014; 134:39-45.

[27] Aguilera JA, Aragón C, Manrique J. Measurement of Stark widths and shifts of Ca II spectral lines. MNRAS 2014; 444:1854-1858.

[28] Wiese WL, Smith MW, Miles BM. Atomic Transition Probabilities. Nat. Stand. Ref. Data Serv., Nat. Bur. Stand, (U.S.) 1969;22.

[29] Kurucz R L, Bell B. Atomic Line Data CD-ROM No 23. Cambridge: Smithsonian Astrophysical Observatory;1995.

[30] Black JH, Weisheit JC, Laviana E. Oscillator strengths and ground-state photoionization cross-sections for $\mathrm{Mg}+$ and $\mathrm{Ca}+$. Astrophys J 1972; 177:567-572. 
Tables

Table 1

Reference Fe II lines used to construct the C $\sigma$ graphs, with their atomic data and typical $k_{t}$ and $\sigma$ / values.

\begin{tabular}{|c|c|c|c|c|c|c|c|c|c|c|c|}
\hline $\begin{array}{l}\lambda \\
(\AA ̊)\end{array}$ & $\begin{array}{l}E_{i} \\
(\mathrm{eV})\end{array}$ & $\begin{array}{l}E_{k} \\
(\mathrm{eV})\end{array}$ & $g_{i}$ & $g_{k}$ & $\begin{array}{l}A_{k i}^{\mathrm{a}} \\
\left(10^{8} \mathrm{~s}^{-1}\right)\end{array}$ & $f^{a}$ & Acc. & $\begin{array}{l}w^{b} \\
(\AA)\end{array}$ & $\begin{array}{l}\text { Acc. } \\
(\%)\end{array}$ & $\begin{array}{l}k_{t}^{\mathrm{c}} \\
\left(10^{-20} \mathrm{~m}^{2} \AA\right)\end{array}$ & $\begin{array}{l}\sigma_{l}^{\mathrm{d}} \\
\left(10^{-20} \mathrm{~m}^{2}\right)\end{array}$ \\
\hline 2331.307 & 0.23 & 5.55 & 10 & 8 & 0.317 & 0.0207 & B & 0.056 & 15 & 0.874 & 15.4 \\
\hline 2338.008 & 0.11 & 5.41 & 4 & 4 & 1.13 & 0.0925 & $\mathrm{~B}+$ & 0.0424 & 15 & 1.74 & 40.6 \\
\hline 2345.339 & 2.63 & 7.92 & 14 & 12 & 0.73 & 0.0520 & $\mathrm{C}$ & 0.038 & 15 & 0.424 & 11.0 \\
\hline 2364.829 & 0.05 & 5.29 & 8 & 8 & 0.59 & 0.0495 & B & 0.042 & 15 & 2.00 & 47.2 \\
\hline 2368.596 & 0.35 & 5.58 & 6 & 4 & 0.606 & 0.0340 & $\mathrm{~B}+$ & 0.047 & 15 & 0.804 & 16.9 \\
\hline 2375.193 & 0.39 & 5.60 & 4 & 2 & 0.981 & 0.0415 & B & 0.051 & 15 & 0.639 & 12.4 \\
\hline 2380.762 & 0.08 & 5.29 & 6 & 8 & 0.31 & 0.0351 & B & 0.039 & - & 1.05 & 26.5 \\
\hline 2391.478 & 0.30 & 5.48 & 8 & 10 & 0.0377 & 0.0041 & B & 0.049 & - & 0.136 & 2.77 \\
\hline $2395.626^{e}$ & 0.05 & 5.22 & 14 & 14 & 1.926 & 0.1661 & $\mathrm{~B}+$ & 0.040 & - & 12.1 & 297 \\
\hline 2404.887 & 0.08 & 5.24 & 6 & 8 & 1.96 & 0.2270 & $\mathrm{~B}+$ & 0.043 & - & 6.91 & 159 \\
\hline 2410.520 & 0.11 & 5.25 & 4 & 6 & 1.55 & 0.2030 & $\mathrm{~B}+$ & 0.043 & 15 & 4.06 & 93.1 \\
\hline 2411.069 & 0.12 & 5.26 & 2 & 2 & 2.37 & 0.2070 & $\mathrm{~B}+$ & 0.038 & 15 & 2.05 & 49.3 \\
\hline 2413.311 & 0.12 & 5.26 & 2 & 4 & 1.02 & 0.1790 & $\mathrm{~B}+$ & 0.039 & 15 & 1.77 & 44.9 \\
\hline 2430.079 & 2.83 & 7.93 & 8 & 10 & 1.91 & 0.2110 & $\mathrm{C}+$ & 0.044 & 15 & 0.898 & 20.2 \\
\hline 2432.262 & 2.84 & 7.94 & 6 & 8 & 1.57 & 0.1860 & $\mathrm{C}+$ & 0.043 & 15 & 0.587 & 13.5 \\
\hline 2439.302 & 3.15 & 8.23 & 12 & 14 & 2.25 & 0.2340 & C & 0.039 & 15 & 1.15 & 29.2 \\
\hline 2444.516 & 2.58 & 7.65 & 6 & 8 & 2.78 & 0.3320 & $C$ & 0.046 & 15 & 1.31 & 28.2 \\
\hline 2585.876 & 0.00 & 4.79 & 10 & 8 & 0.894 & 0.0717 & $\mathrm{~B}+$ & 0.041 & 15 & 4.48 & 108 \\
\hline 2587.945 & 4.15 & 8.94 & 8 & 10 & 1.69 & 0.2130 & C & - & - & 0.341 & 8.44 \\
\hline 2591.543 & 1.04 & 5.82 & 6 & 6 & 0.572 & 0.0576 & B & 0.047 & - & 0.916 & 19.3 \\
\hline 2592.785 & 4.08 & 8.86 & 14 & 16 & 2.74 & 0.3160 & C & 0.045 & 15 & 0.948 & 20.8 \\
\hline 2598.370 & 0.05 & 4.82 & 8 & 6 & 1.43 & 0.1080 & $\mathrm{~B}+$ & 0.039 & - & 5.24 & 131 \\
\hline 2599.396 & 0.00 & 4.77 & 10 & 10 & 2.35 & 0.2390 & $\mathrm{~B}+$ & 0.045 & 15 & 15.1 & 332 \\
\hline 2611.874 & 0.05 & 4.79 & 8 & 8 & 1.2 & 0.1220 & $\mathrm{~B}+$ & 0.0368 & 14 & 5.98 & 161 \\
\hline 2617.618 & 0.08 & 4.82 & 6 & 6 & 0.488 & 0.0501 & $B$ & 0.038 & - & 1.80 & 46.8 \\
\hline
\end{tabular}

aData from Ref. [22].

bStark widths at electron density $N_{e}=10^{17} \mathrm{~cm}^{-3}$ from references [24-26]. When the uncertainty is not displayed in the table, the experimental Stark width is not available, and the average of known Stark widths for lines of the same multiplet has been used. When the Stark width is not provided in the table, an average value of $0.04 \AA$ has been used.

${ }^{\mathrm{c}}$ Calculated for $T=14000 \mathrm{~K}$.

${ }^{\mathrm{d}}$ Calculated for $T=14000 \mathrm{~K}$ and $N_{e}=10^{17} \mathrm{~cm}^{-3}$.

eThe lines at 2395.420 $\AA$ and 2395.626 $\AA$ have been grouped, and the resulting data are indicated. 
Table 2

Ca II lines studied in this work, with their atomic data and typical $k_{t}$ and $\sigma_{\text {I values. }}$

\begin{tabular}{|c|c|c|c|c|c|c|c|c|c|c|c|c|c|c|c|c|}
\hline \multirow[t]{2}{*}{ Line } & \multicolumn{4}{|c|}{ Transition levels } & \multirow{2}{*}{$\begin{array}{l}\lambda_{0} \\
(\AA ̊)\end{array}$} & \multirow{2}{*}{$\begin{array}{l}E_{i} \\
(\mathrm{eV})\end{array}$} & \multirow{2}{*}{$\begin{array}{l}E_{k} \\
(\mathrm{eV})\end{array}$} & \multirow[t]{2}{*}{$g_{i}$} & \multirow[t]{2}{*}{$g_{k}$} & \multirow{2}{*}{$\begin{array}{c}A_{k i}^{\mathrm{a}} \\
\left(10^{8} \mathrm{~s}^{-1}\right)\end{array}$} & \multirow[t]{2}{*}{$f^{\text {a }}$} & \multirow[t]{2}{*}{ Acc. } & \multirow{2}{*}{$\begin{array}{l}w^{b} \\
(\AA ̊)\end{array}$} & \multirow{2}{*}{$\begin{array}{l}\text { Acc. } \\
(\%)\end{array}$} & \multirow{2}{*}{$\begin{array}{l}k_{t}^{\mathrm{c}} \\
\left(10^{-20} \mathrm{~m}^{2} \AA\right)\end{array}$} & \multirow{2}{*}{$\begin{array}{l}\sigma_{l}^{\mathrm{d}} \\
\left(10^{-20} \mathrm{~m}^{2}\right)\end{array}$} \\
\hline & Upper & & Lower & & & & & & & & & & & & & \\
\hline 1 & $3 p^{6} 5 s$ & ${ }^{2} S_{1 / 2}$ & $3 p^{6} 4 p$ & ${ }^{2} \mathrm{p}_{3 / 2}$ & 3736.902 & 3.15 & 6.47 & 4 & 2 & 1.7 & 0.18 & $\mathrm{C}$ & 0.67 & 15 & 12.2 & 18.1 \\
\hline 2 & & & & ${ }^{2} \mathrm{p}^{0}{ }_{1 / 2}$ & 3706.024 & 3.12 & 6.47 & 2 & 2 & 0.88 & 0.18 & $\mathrm{C}$ & 0.66 & 15 & 6.17 & 9.26 \\
\hline 3 & $3 p^{6} 4 d$ & ${ }^{2} D_{5 / 2}$ & $3 p^{6} 4 p$ & ${ }^{2} \mathrm{p}_{3 / 2}$ & 3179.331 & 3.15 & 7.05 & 4 & 6 & 3.6 & 0.82 & $\mathrm{C}$ & 0.49 & 15 & 41.4 & 83.7 \\
\hline 4 & & ${ }^{2} D_{3 / 2}$ & & ${ }^{2} \mathrm{P}_{1 / 2}^{0}$ & 3158.869 & 3.12 & 7.05 & 2 & 4 & 3.1 & 0.93 & $\mathrm{C}$ & 0.53 & 15 & 23.7 & 44.4 \\
\hline 5 & & ${ }^{2} D_{3 / 2}$ & & ${ }^{2} \mathrm{p}_{3 / 2}$ & 3181.275 & 3.15 & 7.05 & 4 & 4 & 0.58 & 0.088 & $\mathrm{C}$ & 0.51 & - & 4.45 & 8.64 \\
\hline 6 & $3 p^{6} 6 s$ & ${ }^{2} S_{1 / 2}$ & $3 p^{6} 4 p$ & ${ }^{2} \mathrm{P}_{3 / 2}$ & 2208.611 & 3.15 & 8.76 & 4 & 2 & 0.62 & 0.023 & $\mathrm{C}$ & 0.73 & 15 & 0.578 & 0.784 \\
\hline 7 & & & & ${ }^{2} \mathrm{p}^{0}{ }_{1 / 2}$ & 2197.787 & 3.12 & 8.76 & 2 & 2 & 0.31 & 0.022 & $\mathrm{C}$ & 0.77 & 15 & 0.280 & 0.360 \\
\hline 8 & $3 p^{6} 5 d$ & ${ }^{2} D_{3 / 2}$ & $3 p^{6} 4 p$ & ${ }^{2} \mathrm{p}_{3 / 2}^{0}$ & 2112.757 & 3.15 & 9.02 & 4 & 6 & 0.97 & 0.097 & $C$ & 0.73 & 15 & 2.23 & 3.03 \\
\hline 9 & & ${ }^{2} D_{5 / 2}$ & & ${ }^{2} \mathrm{p}^{0}{ }_{1 / 2}$ & 2103.235 & 3.12 & 9.02 & 2 & 4 & 0.82 & 0.11 & $\mathrm{C}$ & 0.67 & 15 & 1.29 & 1.90 \\
\hline
\end{tabular}

${ }^{\text {a }}$ Data from Ref. [23].

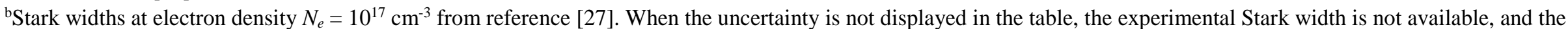
average of known Stark widths for lines of the same multiplet has been used.

${ }^{\mathrm{c}}$ Calculated for $T=14000 \mathrm{~K}$.

${ }^{\mathrm{d}}$ Calculated for $T=14000 \mathrm{~K}$ and $N_{e}=10^{17} \mathrm{~cm}^{-3}$. 
Table 3

Fe and Ca concentrations in the fused glass samples.

\begin{tabular}{lll}
\hline \multirow{2}{*}{ Sample } & \multicolumn{2}{c}{ Concentration $\left(10^{-2}\right.$ at/at $)$} \\
\cline { 2 - 3 } & Fe & Ca \\
\hline A & 0.20 & 0.40 \\
B & 0.034 & 0.070 \\
C & 0.050 & 1.0 \\
\hline
\end{tabular}


Table 4

Values of $\tau / \Delta \lambda_{L}$ for Ca II lines corresponding to samples of different $\mathrm{Ca}$ concentration.

\begin{tabular}{llll}
\hline Line & $\lambda_{0}(\AA)$ & $\tau / \Delta \lambda_{L}(\AA)$ & \\
\cline { 3 - 4 } & & Sample A & Sample B \\
\hline 1 & 3736.902 & 0.11 & 0.020 \\
2 & 3706.024 & 0.057 & 0.010 \\
3 & 3179.331 & 0.38 & 0.066 \\
4 & 3158.869 & 0.22 & 0.038 \\
5 & 3181.275 & 0.041 & 0.007 \\
\hline
\end{tabular}


Table 5

Experimental transition probabilities of Ca II determined in this work, compared to experimental and theoretical values reported in the literature.

\begin{tabular}{|c|c|c|c|c|c|c|c|c|c|c|c|c|}
\hline \multirow{2}{*}{\multicolumn{2}{|c|}{$\begin{array}{l}\text { Transition levels } \\
\text { Upper }\end{array}$}} & \multirow{3}{*}{ Lower } & & \multirow[t]{3}{*}{$\lambda(\AA)$} & \multicolumn{8}{|c|}{ Transition probabilities $\left(10^{8} \mathrm{~s}^{-1}\right)$} \\
\hline & & & & & \multicolumn{2}{|l|}{ Experiments } & \multicolumn{6}{|l|}{ Theory } \\
\hline & & & & & This work & Ref. [8] & Ref. [28] & Ref. [12] & Ref. [29] & Ref. [14] & Ref. [16] & Ref. [23] \\
\hline \multirow[t]{2}{*}{$3 p^{6} 5 s$} & ${ }^{2} \mathrm{~S}_{1 / 2}$ & $3 p^{6} 4 p$ & ${ }^{2} \mathrm{P}^{0}{ }_{3 / 2}$ & 3736.902 & $1.57 \pm 0.11$ & $1.5 \pm 0.2$ & 1.65 & 1.61 & 1.701 & 1.687 & 1.705 & 1.7 \\
\hline & ${ }^{2} \mathrm{~S}_{1 / 2}$ & & ${ }^{2} \mathrm{P}_{1 / 2}^{0}$ & 3706.024 & $0.837 \pm 0.059$ & $0.8 \pm 0.1$ & 0.84 & 0.81 & 0.8670 & 0.8456 & 0.8550 & 0.88 \\
\hline \multirow[t]{3}{*}{$3 p^{6} 4 d$} & ${ }^{2} \mathrm{D}_{5 / 2}$ & $3 p^{6} 4 p$ & ${ }^{2} \mathrm{P}_{3 / 2}^{0}$ & 3179.331 & $3.44 \pm 0.24$ & $3.2 \pm 0.2$ & 3.59 & & 3.573 & 3.396 & 3.506 & 3.6 \\
\hline & ${ }^{2} \mathrm{D}_{3 / 2}$ & & ${ }^{2} \mathrm{P}_{1 / 2}^{0}$ & 3158.869 & $2.83 \pm 0.20$ & $3.0 \pm 0.2$ & 3.05 & & 2.984 & 2.851 & 2.944 & 3.1 \\
\hline & ${ }^{2} D_{3 / 2}$ & & ${ }^{2} \mathrm{P}_{3 / 2}^{0}$ & 3181.275 & $0.71 \pm 0.09$ & & 0.60 & & 0.5870 & 0.5659 & 0.5843 & 0.58 \\
\hline \multirow[t]{2}{*}{$3 p^{6} 6 s$} & ${ }^{2} \mathrm{~S}_{1 / 2}$ & $3 p^{6} 4 p$ & ${ }^{2} \mathrm{P}^{0}{ }_{3 / 2}$ & 2208.611 & $0.396 \pm 0.048$ & & 0.62 & 0.58 & 0.6146 & 0.6440 & 0.6416 & 0.62 \\
\hline & ${ }^{2} \mathrm{~S}_{1 / 2}$ & & ${ }^{2} \mathrm{P}_{1 / 2}^{0}$ & 2197.787 & $0.208 \pm 0.025$ & & 0.313 & 0.29 & 0.3111 & 0.3237 & 0.3226 & 0.31 \\
\hline \multirow[t]{2}{*}{$3 p^{6} 5 d$} & ${ }^{2} \mathrm{D}_{5 / 2}$ & $3 p^{6} 4 p$ & ${ }^{2} \mathrm{P}_{3 / 2}^{0}$ & 2112.757 & $0.593 \pm 0.071$ & $1.4 \pm 0.2^{*}$ & 1.10 & & 0.9817 & 1.012 & 0.9580 & 0.97 \\
\hline & ${ }^{2} \mathrm{D}_{3 / 2}$ & & ${ }^{2} \mathrm{P}_{1 / 2}^{0}$ & 2103.235 & $0.421 \pm 0.051$ & & 0.93 & & 0.8166 & 0.8550 & 0.8102 & 0.82 \\
\hline
\end{tabular}

* Lines at $2112.757 \AA$ And $2113.146 \AA$ not resolved. 
Table 6

Experimental statistically-weighted transition probabilities of Ca II determined in this work, compared to theoretical values reported in the literature.

\begin{tabular}{|c|c|c|c|c|c|c|c|c|c|c|}
\hline \multicolumn{4}{|c|}{ Transition levels } & \multirow[t]{3}{*}{$\lambda(\AA)$} & \multicolumn{6}{|c|}{ Transition probabilities $\left(10^{8} \mathrm{~s}^{-1}\right)$} \\
\hline \multirow[t]{2}{*}{ Upper } & & \multirow{2}{*}{\multicolumn{2}{|c|}{ Lower }} & & \multirow{2}{*}{$\begin{array}{l}\text { Experiment } \\
\text { This work }\end{array}$} & \multicolumn{5}{|l|}{ Theory } \\
\hline & & & & & & Ref. [28] & Ref. [30] & Ref. [11] & Ref. [12] & Ref. [15] \\
\hline $3 p^{6} 5 s$ & ${ }^{2} S$ & $3 p^{6} 4 p$ & ${ }^{2} \mathrm{P}^{0}$ & 3726.5 & $2.407 \pm 0.17$ & 2.49 & 2.60 & 2.428 & & 2.582 \\
\hline $3 p^{6} 4 d$ & ${ }^{2} \mathrm{D}$ & $3 p^{6} 4 p$ & ${ }^{2} \mathrm{P}^{0}$ & 3172.6 & $3.480 \pm 0.26$ & 3.62 & 3.58 & 3.297 & 3.25 & 3.453 \\
\hline $3 p^{6} 6 s$ & ${ }^{2} S$ & $3 p^{6} 4 p$ & ${ }^{2} \mathrm{P}$ & 2205.0 & $0.604 \pm 0.073$ & 0.93 & 0.935 & 0.8845 & & \\
\hline $3 p^{6} 5 d$ & ${ }^{2} \mathrm{D}$ & $3 p^{6} 4 p$ & ${ }^{2} \mathrm{P}^{0}$ & 2109.6 & $0.524 \pm 0.063$ & 1.10 & 0.982 & 0.8049 & 1.00 & \\
\hline
\end{tabular}


Figures

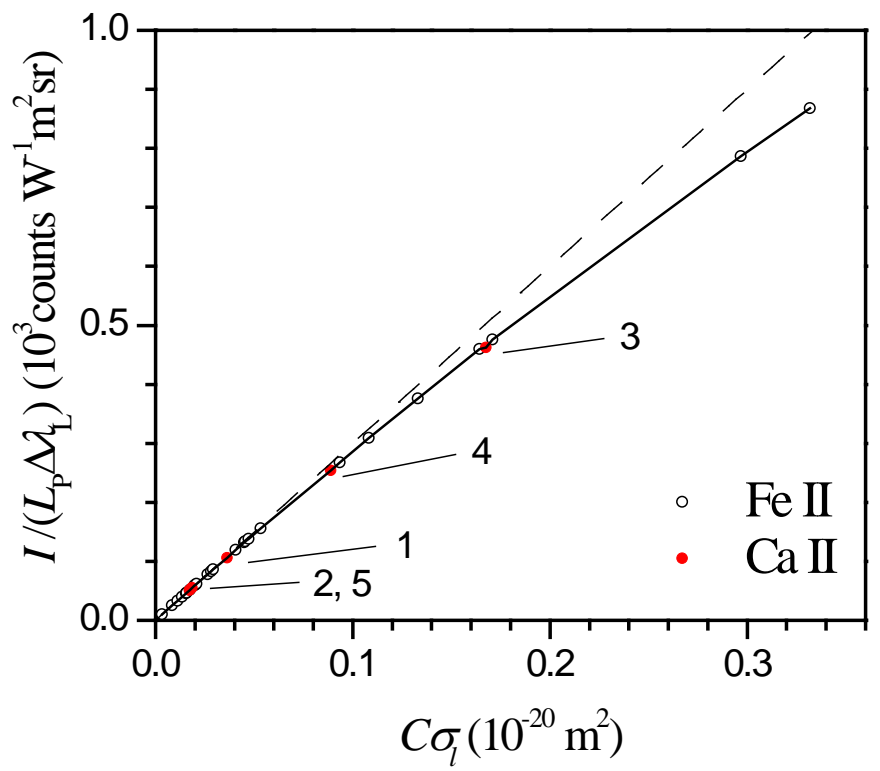

Fig. 1. Example of theoretical $C \sigma$ graph including the reference Fe II lines and the more intense Ca II lines studied. The Ca II lines are numbered according to Table 2. The values of the plasma parameters used in the calculation are $T=14000 \mathrm{~K}, N_{e}$ $=10^{17} \mathrm{~cm}^{-3}, \mathrm{Nl}=3 \times 10^{20} \mathrm{~m}^{-2}, \beta A=10^{6}$ counts $\mathrm{W}^{-1} \mathrm{~m}^{2} \mathrm{sr}$, and the concentrations in the sample are 0.001 at/at for Fe and 0.002 at/at for Ca. 


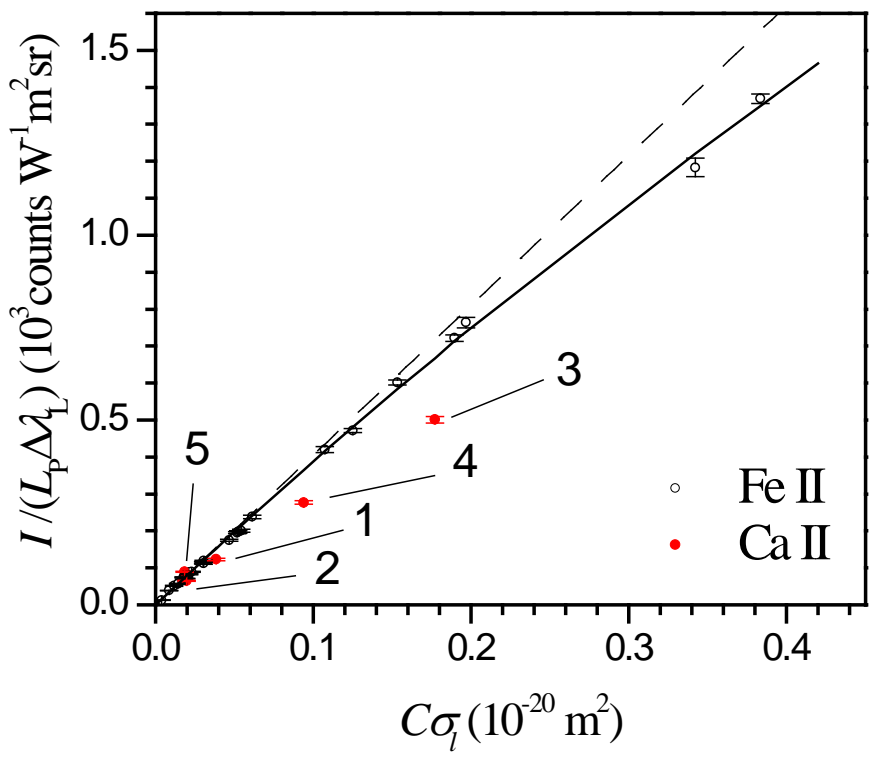

Fig. 2. Experimental $C \sigma$ graph obtained using sample A (see Table 3), including the reference Fe II lines and the more intense Ca II lines studied. The Ca II lines are numbered according to Table 2. The solid line is the fitting of the Fe II data to a $C \sigma$ curve and the dashed line is the linear limit of this curve. 


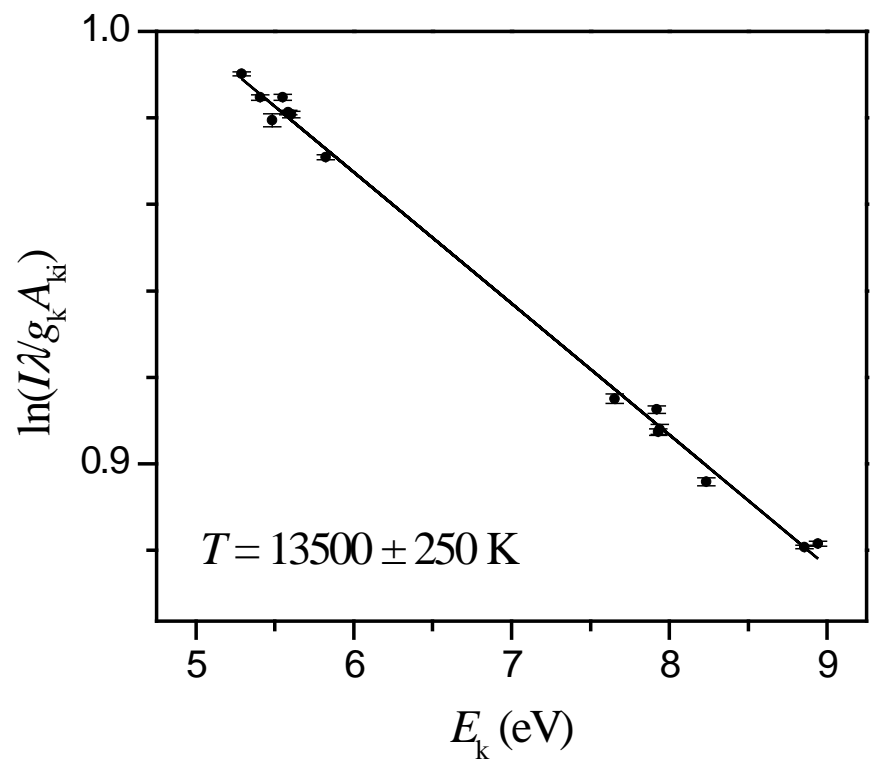

Fig. 3. Boltzmann plot constructed from the Fe II lines with negligible selfabsorption. The resulting temperature is indicated. 

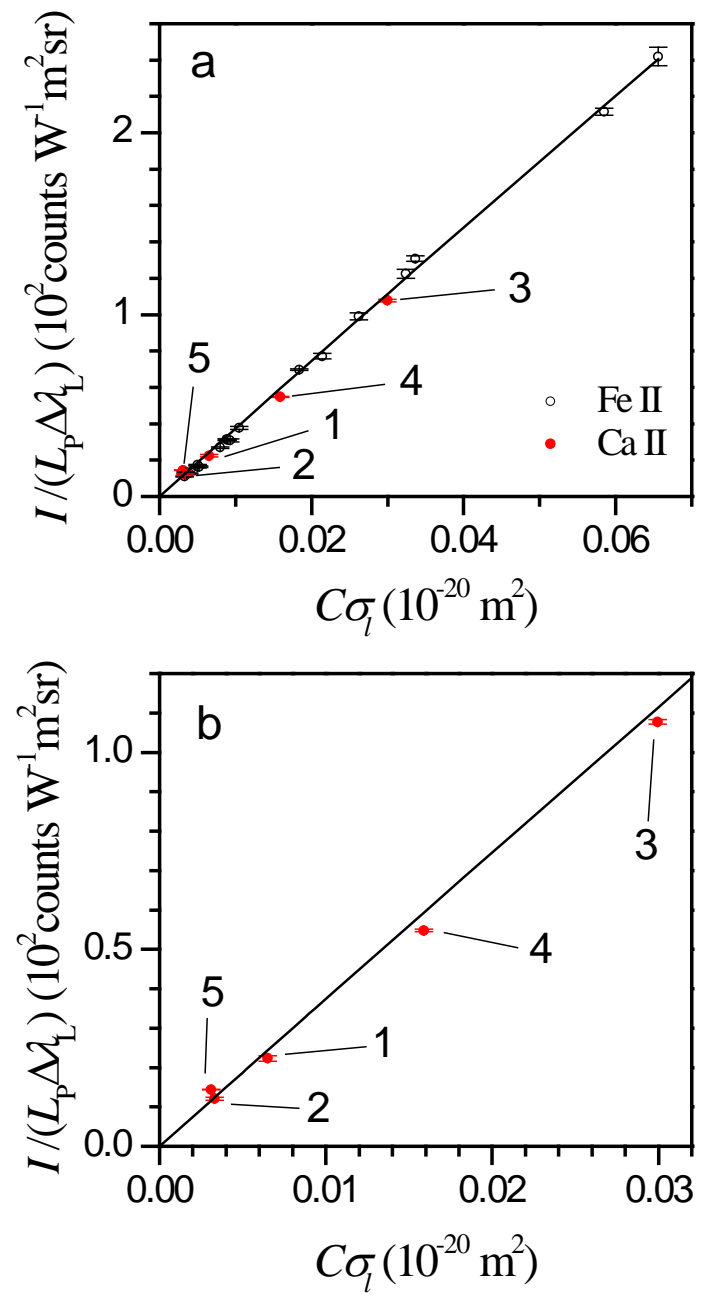

Fig. 4. (a) Experimental $C \sigma$ graph obtained using sample B (see Table 3), including the reference Fe II lines and the more intense Ca II lines studied. The Ca II lines are numbered according to Table 2. The solid line is the fitting of the Fe II data to a $C \sigma$ curve which is linear in this case. (b) Enlargement showing the $C \sigma$ curve and the Ca II data. 